\section{¿Está indicada la profilaxis antibiótica con ciprofloxacino en la realización de cistoscopia flexible?}

\author{
MARÍA DEL CARMEN CANO-GARCÍA ${ }^{1}$, ROSARIO CASARES-PÉREZ ${ }^{1}$, \\ ELISABETH CASTILLO-GALLARDO ${ }^{1}$, SERGIO MERINO-SALAS ${ }^{2}$, \\ MIGUEL ARRABAL-MARTÍN ${ }^{3, a}$, MIGUEL ÁNGEL ARRABAL-POLO ${ }^{1, a}$
}

\section{Usefulness of antimicrobial prophylaxis with ciprofloxacin prior to flexible cystoscopy}

Background: Flexible cystoscopy is a common test in clinical practice done with or without antibiotic prophylaxis. Aim: To evaluate the efficacy of antibiotic prophylaxis with ciprofloxacin to reduce the incidence of urinary infection. Material and Methods: Prospective, non-randomized observational study that included 60 patients divided into two groups. Group 1 received prophylactic ciprofloxacin $500 \mathrm{mg}$, one hour prior to the procedure and group 2 did not receive prophylaxis. The presence of bacteriuria, symptoms or signs of urinary infection or attending Emergency rooms or primary care for these symptoms were recorded during the seven days after the cystoscopy. Results: In groups 1 and 2, four and one patients had a positive urine culture, respectively. Only one patient in group 1 consulted in primary care for symptoms. No significant differences in symptoms or signs of urinary infection between groups were observed. Conclusions: In this group of patients, antibiotic prophylaxis with ciprofloxacin $500 \mathrm{mg}$ prior to cystoscopy had no benefit.

(Rev Med Chile 2015; 143: 1001-1004)

Key words: Anti-bacterial agents; Ciprofloxacin; Cytoscopy; Prevention and control.

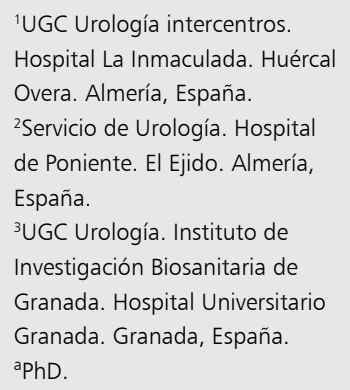

Los autores declaran que no tienen conflicto de intereses. No existe financiación de este artículo ni de la investigación.

Recibido el 2 de enero de 2015, aceptado el 11 de junio de 2015 .

Correspondencia a:

Miguel Ángel Arrabal-Polo. C/Camino de Ronda, 143, 4F. Granada. CP: 18003. Teléfono: 34628837188 arrabalp@ono.com

\section{L} a cistoscopia flexible es una técnica ambulatoria muy útil en el diagnóstico y seguimiento de la patología del tracto urinario inferior en la que se valora la uretra y la vejiga masculina y femenina, realizándose el procedimiento en la mayoría de los casos con anestesia local. Esta técnica supuso una revolución en el área diagnóstica en urología, ya que se sustituyó la realización de cistoscopia rígida en la mayoría de los centros hospitalarios por cistoscopia flexible ${ }^{1}$. Aunque el procedimiento diagnóstico con cistoscopia puede realizarse usando cistoscopio rígido o flexible, como hemos referido previamente, en los hombres es preferible el uso de los instrumentos flexibles porque son mejor tolerados, causando menos dolor y menos complicaciones ${ }^{2}$, sin embargo, en las mujeres el uso indistinto de la cistoscopia flexible o rígida es bien tolerada, con buenos resultados en términos de dolor y eficacia, debiéndose usar una $\mathrm{u}$ otra en función de las preferencias del urólogo y de la paciente ${ }^{3}$. En líneas generales, se considera que la cistoscopia no se asocia con una elevada percepción de dolor, siendo en cualquier caso habitualmente mayor en el primer procedimiento y disminuyendo en los posteriores, de tal manera que es tolerada tanto en hombres como en mujeres con anestesia local ${ }^{4}$. En la actualidad, aunque la Guía Europea de Urología ${ }^{5}$ recomienda únicamente profilaxis antibiótica en pacientes de alto riesgo, los protocolos en cada hospital son diferentes y todavía sigue haciéndose profilaxis para disminuir el riesgo de bacteriuria e incluso existen 
estudios que afirman que el uso de antibióticos previene o disminuye la bacteriuria ${ }^{6}$.

El objetivo de este estudio es comparar la existencia de bacteriuria y síntomas de infección urinaria en pacientes sometidos a cistoscopia flexible con ciprofloxacino, frente a no tomar profilaxis.

\section{Material y Método}

Sesenta pacientes son incluidos en un estudio observacional prospectivo no aleatorizado, en el que 2 urólogos diferentes realizan la cistoscopia usando uno de ellos profilaxis antibiótica y otro sin usar dicha profilaxis, según la práctica clínica habitual de cada uno. Previamente a la inclusión en el estudio, se comprueba con urocultivo 3 días antes de la prueba la ausencia de infección urinaria. Los pacientes son divididos en dos grupos:

- Grupo 1:30 pacientes sometidos a cistoscopia flexible que toman 1 hora antes ciprofloxacino $500 \mathrm{mg}$.

- Grupo 2:30 pacientes sometidos a cistoscopia flexible sin profilaxis antibiótica.

\section{Criterios de inclusión}

Hombres y mujeres mayores de 18 años a los que se indica cistoscopia flexible según práctica clínica habitual.

\section{Criterios de exclusión}

Pacientes con sonda vesical o talla suprapúbica, pacientes que han tenido infección urinaria en el último mes, pacientes portadores de catéter ureteral o sonda de nefrostomía, pacientes con estenosis de uretra.

\section{Variables principales analizadas}

Síntomas de infección urinaria durante los 7 días posteriores a la cistoscopia, asistencia a urgencias o atención primaria por síntomas de infección, bacteriuria (mediante urocultivo a los 7 días), tipo de microorganismo responsable en los casos de infección.

\section{Variables secundarias}

Edad, sexo, motivo de la cistoscopia, resultado de la cistoscopia.

\section{Estudio estadístico}

Se estudiará en primer lugar las proporciones y medias de las variables analizadas en el estudio $y$, posteriormente, se realizará estudio estadístico de los resultados aplicando test-t para análisis de variables continuas y test de $\chi^{2}$ para análisis de variables cualitativas. Se comprueba normalidad de las variables aplicando test Kolmogorov-Smirnov y análisis de la varianza con test de Levene. Consideramos significación estadística si $\mathrm{p} \leq 0,05$. Análisis realizado con programa SPSS 17.0 para Windows.

\section{Aspectos éticos}

Todos los pacientes fueron informados acerca del estudio y dieron su consentimiento informado para participar en él. El Comité de Ética de nuestra Área de Salud aprobó la realización del estudio.

\section{Resultados}

La edad media de los pacientes fue de 66.96 $\pm 12,52$ años en el grupo 1 versus 63,96 $\pm 11,59$ años en el grupo $2(\mathrm{p}=0,36)$. En el grupo 1,19 hombres y 11 mujeres y en el grupo 2, 22 hombres y 8 mujeres $(p=0,1)$. El motivo más frecuente de solicitar cistoscopia fue revisión de cáncer vesical superficial previamente intervenido, en $66,6 \%$ en el grupo 1 y en $50 \%$ en el grupo 2 . Otras causas menos frecuentes fueron hematuria y síntomas del tracto urinario inferior (Tabla 1). En $70 \%$ de los casos en el grupo 1 la cistoscopia fue normal, mientras que en el grupo 2 fue normal en $30 \%$ de casos (Tabla 2). Al analizar las variables correspondientes a la presencia de síntomas-signos de infección urinaria, observamos que ningún paciente ni del grupo 1 o grupo 2 tuvo que acudir a Urgencias en los 7 días posteriores a la cistoscopia y que únicamente 1 paciente del grupo 1 acudió a la consulta de Atención Primaria. Sólo 5 pacientes del grupo 1 y 8 pacientes del grupo 2 presentaron síntomas del tracto urinario durante los 7 días posteriores a la cistoscopia, sin diferencias significativas en este parámetro (Figura 1). En relación con la presencia de bacteriuria, el urocultivo fue positivo en 4 pacientes del grupo $1 \mathrm{y}$ en 1 paciente del grupo 2 . No se han observado diferencias estadísticamente significativas en ninguno de los parámetros analizados (Figura 1). En los 4 pacientes del grupo 1 con urocultivo positivo los microorganismos encontrados fueron Escherichia coli, Proteus mirabilis, Klebsiella pneumonia y Enterococo faecalis, mientras que en el único paciente del grupo 1 con 
Tabla 1. Causas que motivaron la solicitud de cistoscopia en los grupos de estudio usando o no profilaxis antibiótica

\begin{tabular}{|c|c|c|}
\hline & Grupo 1 & Grupo 2 \\
\hline Revisión tumor vesical & $66,6 \%(20)$ & $50 \%(15)$ \\
\hline Hematuria & $10 \% \quad$ (3) & $20 \% \quad(6)$ \\
\hline Síndrome miccional & $16,6 \% \quad(5)$ & $10 \% \quad(3)$ \\
\hline Otros & $6,8 \% \quad(2)$ & $20 \% \quad(6)$ \\
\hline
\end{tabular}

$\mathrm{p}=0,1$ tras test $\chi^{2}$.
Tabla 2. Resultados de la cistoscopia flexible en los grupos de estudio

\begin{tabular}{|llcc|}
\hline & Grupo 1 & \multicolumn{3}{c|}{ Grupo 2 } \\
\hline Normal & $70 \% \quad(21)$ & $30 \%$ & $(9)$ \\
\hline Tumor & $13,3 \%(4)$ & $20 \%$ & $(6)$ \\
\hline Vejiga de lucha & $13,3 \%(4)$ & $3,4 \%$ & $(1)$ \\
Otros & $3,4 \%(1)$ & $56,6 \%(14)$ \\
\hline
\end{tabular}

$\mathrm{p}=0,003$ tras test $\chi^{2}$.

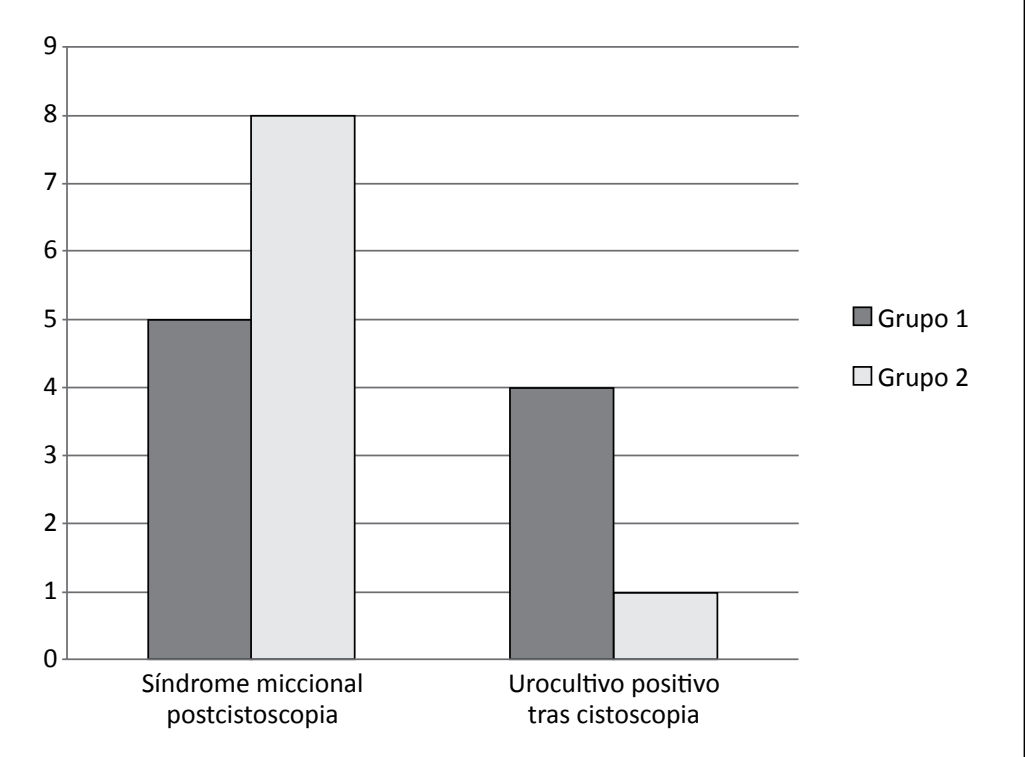

Figura 1. Diferencia entre grupos en la aparición de síndrome miccional y urocultivo positivo tras cistoscopia. urocultivo positivo el microorganismo aislado fue Escherichia coli.

\section{Discusión}

Como hemos comentado anteriormente, aunque la guía clínica de la Asociación Europea de Urología ${ }^{5}$ recomienda actualmente el uso de profilaxis antibiótica en pacientes de alto riesgo (diabéticos, inmunodeprimidos), sigue usándose la profilaxis antibiótica de forma arbitraria en los diferentes hospitales donde se realiza esta técnica. La infección urinaria y la presencia de bacteriuria es poco frecuente tras la cistoscopia flexible ${ }^{7} \mathrm{y}$ existe una baja incidencia de bacteriuria ${ }^{8}$, a pesar de lo cual, diferentes autores analizan el papel de la profilaxis antibiótica en la realización de este procedimiento. Alguno de esos estudios defienden que el uso de ciprofloxacino como profilaxis reduce significativamente la bacteriuria frente a placebo y a trimetoprim ${ }^{6}$, mientras que otros no observan diferencias en el uso o no de profilaxis antibiótica ${ }^{9,10}$. El estudio de Johnson et $\mathrm{al}^{6}$ demuestra que la administración de 1 hora antes de la cistoscopia flexible de $500 \mathrm{mg}$ de ciprofloxacino reduce de forma significativa la bacteriuria frente a placebo y frente a trimetoprim. En nuestro estudio observamos que ciprofloxacino $500 \mathrm{mg}$ administrado una hora antes del procedimiento ni reduce ni evita la aparición de síntomas de infección urinaria, bacteriuria o asistencia a 
urgencias/atención primaria por este motivo, por lo que no coincidimos con la afirmación de Johnson. El estudio de Jiménez et $\mathrm{al}^{9} \mathrm{y}$ de Herr et $\mathrm{al}^{10}$ demuestran que no existe un beneficio sobre la infección urinaria la administración de profilaxis antibiótica. Nuestro estudio coincide más en la línea de estos dos últimos trabajos en los que no se observa beneficio alguno de la administración de profilaxis antibiótica. No obstante, este sigue siendo un tema a discusión, puesto que las características epidemiológicas y microbiológicas de cada área de salud pueden influir en la toma de la decisión y en los resultados posteriores. Como conclusión de nuestro estudio, no recomendamos el uso de antibiótico profiláctico (en este caso ciprofloxacino) en la realización de cistoscopia flexible, puesto que no ofrece beneficio alguno.

Agradecimientos: Este trabajo forma parte de la Tesis Doctoral de María del Carmen Cano-García, dentro del Doctorado de Medicina Clínica y Salud Pública de la UGR y cuyos directores son Miguel Ángel Arrabal Polo y Miguel Arrabal Martín.

\section{Referencias}

1. Pillai PL, Sooriakumaran P. Flexible cystoscopy: a revolution in urological practice. $\mathrm{Br} \mathrm{J}$ Hosp Med (London). 2009; 70: 583-5.

2. Ciclone A, Cantiello F, Damiano R. Cystoscopy in non-muscle-invasive bladder cancer: when and how (rigid or flexible). Urología 2013; 80: 11-5.

3. Gee JR, Waterman BJ, Jarrard DF, Hedican SP, Bruskewitz RC, Nakada SY. Flexible and rigid cystoscopy in women. JSLS. 2009; 13: 135-8.

4. Greenstein A, Greenstein I, Senderovich S, Mabjeesh NJ. Is diagnostic cystoscopy painful? Analysis of 1,320 consecutive patients. Int Braz J Urol. 2014; 40: 533-8.

5. Guidelines on Urological Infections. http://www. uroweb.org/gls/pdf/19\%20 Urological\%20infections_ LR.pdf.

6. Johnson MI, Merrilees D, Robson WA, Lennon T, Masters J, Orr KE, et al. Oral ciprofloxacin or trimethroprim reduces bacteriuria after flexible cystoscopy. BJU Int 2007; 100: 826-9.

7. Wilson L, Ryan J, Thelning C, Masters J, Tuckey J. Is antibiotic prophylaxis required for flexible cystoscopy? A truncated randomized double-blind controlled trial. J Endourol 2005; 19: 1006-8.

8. Lo TS, Khanuengkitkong S, Tan YL, Dass AK, Wu PY, Al-Kharabsheh AM. Low incidence of bacteriuria with outpatient flexible cystoscopy. Aust N Z J Obstet Gynaecol 2014; 54: 366-70.

9. Jiménez Pacheco A, Lardelli Claret P, López Luque A, Lahoz-García C, Arrabal Polo MA, Nogueras Ocaña M. Ensayo clínico aleatorizado sobre profilaxis antibiótica en la uretrocistoscopia flexible. Arch Esp Urol 2012; 65: 542-9.

10. Herr HW. Should antibiotics be given prior to outpatient cystoscopy? A plea to urologist to practice antibiotic stewardship. Eur Urol 2014; 65: 839-42. 